\title{
Autoconsciência da aparência e a adaptação no ensino superior: Estudo exploratório
}

\author{
Self-consciousness of appearance and adaptation in higher education: Exploratory study
}

Artigo Original | Original Article

\section{José Mendes PhD (1a), Adriana Fraga Estudante Psy (2b), Carla Medeiros Estudante Psy (2c), Daniela Moniz Estudante Psy (2c), Luana Miranda Estudante Psy (2c), Teresa Medeiros PhD (2,3d)}

(1) INTELECTO - Psicologia \& Investigação, Ponta Delgada, Açores, Portugal

(2) Universidade dos Açores, Ponta Delgada, Açores, Portugal

(3) Instituto de Psicologia Cognitiva, Desenvolvimento Social e Humano da Faculdade de Psicologia da Universidade de Coimbra, Portugal

(a) Supervisão do estudo; Revisão de literatura; Análise estatística; Redação e revisão do manuscrito.

(b) Recolha de dados; Revisão de literatura.

(c) Recolha de dados; Revisão de literatura; Redação do manuscrito.

(d) Coordenadora do estudo; Redação e revisão integral do artigo

Autor para correspondência | Corresponding author: José C. S. Mendes; Rua do Monte, 52B, Fração L, r/c esq. nascente, $9500-451$ Ponta Delgada, Portugal; josemendes@intelecto.pt

\section{RESUMO}

\section{Palavras-Chave}

Adultos emergentes

Adaptação Ensino Superior

Autoconsciência da

Aparência

Estudo exploratório
Contexto: A Psicologia da Aparência tem merecido pouca atenção dos investigadores portugueses. As representações contemporâneas do corpo ideal (magro, atlético e com formas), muito avolumadas pelos meios de comunicação social e redes sociais, criam frequentemente a insatisfação com a aparência. Estudos recentes referem que os estudantes universitários se encontram insatisfeitos com a imagem corporal.

Objetivo: Avaliar possíveis relações entre as preocupações com aparência, nomeadamente a autoconsciência da aparência, e a adaptação ao Ensino Superior por parte de estudantes portugueses.

Método: Exploratório e quantitativo. Participaram 206 estudantes do Ensino Superior, tendo respondido a um Questionário Sociodemográfico, à versão portuguesa reduzida da Escala de Avaliação da Aparência de Derriford (DAS-14) e ao Questionário de Adaptação ao Ensino Superior (QAES).

Resultados: Verificaram-se diferenças significativas na DAS-14 (autoconsciência da aparência) entre os sexos; correlação moderada entre a DAS-14 e a dimensão adaptação pessoal-emocional e relações fracas entre a DAS14 e as restantes dimensões do QAES (adaptação interpessoal, adaptação à instituição, adaptação académica, compromisso com o curso e desenvolvimento de carreira). A autoconsciência da aparência apresenta-se quer como variável preditora, quer como variável de resposta na dimensão adaptação pessoal-emocional.

Conclusão: Existe relação entre o sexo a autoconsciência da aparência. Os estudantes que têm menor concentração nos sentimentos negativos no corpo têm uma maior relação com as dimensões sociais, cognitivas e contextuais da adaptação ao ensino superior. As preocupações com a aparência e a adaptação pessoalemocional influenciam-se mutuamente, isto é, a aceitação da aparência parece ser relevante para a adaptação e para o desenvolvimento da identidade dos adultos emergentes. O estudo abre futuras investigações na área da Psicologia da Aparência.

\section{ABSTRACT}

\section{Keywords}

Emerging Adulthood Higher Education Adaptation Self-consciousness of Appearance Exploratory study
Background: Portuguese researchers have not devoted too much attention to Psychology of Appearance. Contemporary representations of the ideal body (thin, athletic, with defined muscles), widely disseminated by traditional media and by social media, often create dissatisfaction with appearance. Recent studies indicate that university students find themselves dissatisfied with their body image.

Aim: To assess possible relationships between appearance concerns, especially self-consciousness of appearance, and adaptation to higher education by Portuguese students.

Method: Exploratory and quantitative. Two hundred and six (206) students answered a sociodemographic questionnaire, the Portuguese short version of the Derriford Appearance Scale (DAS-14) and the Higher Education Adaptation Questionnaire (QAES).

Results: There were significant differences in the DAS-14 between sexes; a moderate correlation between DAS14 (self-consciousness of appearance) and personal and emotional adaptation; and weak relationships between self-consciousness of appearance and the remaining dimensions of QAES (interpersonal adaptation, institution adaptation, academic adaptation, commitment to the course, and career development). The self-consciousness of appearance presents itself as both a predictor variable and a response variable in the personal-emotional adaptation dimension.

Conclusions: There seems to be a relationship between sex and self-consciousness of appearance: the lower the concentration of negative feelings around how the body looks the higher the adaptation to higher education along with social, cognitive and contextual dimensions. On the other hand, adaptation to higher education seems to influence the self- consciousness of appearance in emergent adults, with greater emphasis on personalemotional adaptation. This study allows us to suggest future investigations in the evaluation of selfconsciousness of appearance as a predictor variable. 


\section{INTRODUÇÃO}

As representações do corpo têm sofrido alterações ao longo da História. No decorrer do século XX, particularmente na segunda metade da centúria, enaltece-se um ideal de corpo que se quer/deseja magro, atlético e com formas definidas, e que se passa a constituir como um objeto de consumo perante a oferta de produtos e serviços num mercado crescente (Rodrigues, Teves, \& Medeiros, 2013). A sociedade, muito através dos órgãos de comunicação social, sobretudo a televisão, releva uma cultura que elege o corpo como uma fonte de identidade; há a procura por uma figura "perfeita", que conduz ao afastamento do corpo real (Martins, Nunes, \& Noronha, 2008). Este corpo ideal está diretamente associado à imagem de poder, beleza e mobilidade social, sendo crescente a insatisfação das pessoas com a própria aparência (Rodrigues et al., 2013).

As representações do corpo parecem fazer parte integrante do processo de adaptação ao ensino superior. De facto, o processo de adaptação ao ensino superior requer um conjunto de competências adaptativas a um novo contexto, mobilizando tanto os recursos pessoais e contextuais (desafios emocionais, académicos, sociais e institucionais), como o desenvolvimento de novas estruturas de funcionamento interpessoal (Freitas, Raposo, \& Almeida, 2007; Vieira \& Grantham, 2011). No entanto, os estudantes do ensino superior, apesar de apresentarem experiências e vivências diversas, apresentam características comuns (Souza \& Alvarenga, 2016), onde a adaptação ao ensino superior é modulada, do ponto de vista psicossocial, por fatores pessoais, académicos e sociais (Deaño, Gil, Rodríguez, García-Señorán, \& González, 2014). Estudos referem que a maioria dos adultos emergentes que frequenta o ensino superior sente insatisfação com a sua imagem corporal (e.g., Behmani \& Kumar, 2016; Souza \& Alvarenga, 2016). Verifica-se, assim, que as questões de identidade corporal e de (in)satisfação com corpo, que estavam muito circunscritas à puberdade e à adolescência, foram expandidas para a adultez emergente, período de desenvolvimento que caracteriza os estudantes tradicionais do ensino superior (Arnett, 2004).

Para melhor compreensão sobre as preocupações com a aparência, considera-se relevante diferenciar os conceitos de Imagem Corporal, Insatisfação com a Imagem Corporal e Autoconsciência da Aparência. O conceito de Imagem Corporal é multidimensional, englobando perceções, crenças, pensamentos, sentimentos e comportamentos relativos ao self físico (physical self; MacKenzie, 2012). Não existindo uma definição sobre uma imagem corporal negativa (Cash, 2002), a Insatisfação com a Imagem Corporal revela-se um conceito mais amplo, constituído por pensamentos e sentimentos negativos que o indivíduo tem sobre os aspetos não visíveis do corpo, influenciado por um inúmero de variáveis biopsicossociais (Cash \& Smolak, 2011; Latiff, Muhamad, \& Rahman, 2018; Smolak, 2012). Por último, a Autoconsciência da Aparência, concentra-se nos sentimentos negativos em torno do aspeto do corpo (Moss \& Rosser, 2012), isto é, como o indivíduo se sente e se comporta relativamente a algum aspeto incomodativo da sua aparência (Carr, Moss, \& Harris, 2005).

De acordo com Cash e Pruzinsky (2002), a imagem corporal tem um efeito significativo sobre o que as pessoas sentem e pensam sobre si próprias, fazendo com que comportamento do indivíduo e o relacionamento social se influenciem. Uma imagem corporal negativa pode atingir o funcionamento social e a qualidade de vida no quotidiano dos indivíduos (Rumsey \& Harcourt, 2012), podendo o impacto e aceitação das relações interpessoais influenciar a construção da imagem corporal (Tiggemann, 2015). Nestes processos a sociedade desempenha um papel importante na modelação do corpo através das relações sociais e culturais (Lopes, Mendes, \& Sousa, 2017).

A aparência tem uma importância representativa nos valores sociais, devendo analisar-se a aparência, quer pela perspetiva exterior/sociedade, quer pela perspetiva interior/indivíduo (Rumsey \& Harcourt, 2012).

Em face dos vários estudos já referidos que apontam a tendência para: i) a insatisfação dos estudantes do ensino superior em relação ao seu próprio corpo; ii) a aparência desempenhar um papel relevante na interação social (Carr, Harris, \& James, 2000) e iii) as comparações com a aparência serem um fator sociocultural importante na perceção da imagem corporal (Fardouly, Pinkus, \& Vartanian, 2017), existe a necessidade de maior investigação sobre a avaliação da autoconsciência da aparência em contexto universitário (Medeiros et al., 2018).

Assim, contribuindo para este desiderato, pretendese com a presente investigação compreender as relações entre a adaptação ao ensino superior e as preocupações que os estudantes universitários adultos emergentes - sentem em relação à sua aparência. 


\section{MÉTODO}

\section{Procedimentos}

Após a apreciação dos instrumentos mais adequados para a medição das variáveis em estudo, optou-se pelo uso de instrumentos já validados. Assim, depois da explicitação do âmbito do estudo e dos objetivos, obtevese a autorização dos autores dos instrumentos abaixo referidos para a utilização neste estudo. Posteriormente, selecionado o universo dos estudantes do ensino superior português, procedeu-se à clarificação dos objetivos e às condições de investigação e entregou-se o Termo de Consentimento Livre e Esclarecido que foi preenchido por cada participante do estudo, dando assim a sua anuência, garantidos que foram o anonimato e a confidencialidade individual. Para a recolha de dados, procedeu-se à impressão dos instrumentos em formato papel para aplicação presencial, tendo-se disponibilizado igualmente o estudo através de uma plataforma digital (Goog/e Forms), em que os participantes teriam de ler o Termo de Consentimento Livre e Esclarecido, aceitando a participação na presente investigação.

\section{Participantes}

Participaram neste estudo, 206 adultos emergentes que frequentam o ensino superior, distribuídos por 12 instituições, com a seguinte distribuição: distrito do Porto (22,8\%), Lisboa (18\%), Coimbra (13,6\%), Açores (11,2\%) e Minho (10,7\%), Aveiro (9,2\%), estando os restantes participantes distribuídos pelos concelhos Guarda (3,4\%), Algarve, Setúbal e Évora (2,9\%), Viseu (1,5\%) e Covilhã (1\%). A amostra foi constituída por 35 participantes do sexo masculino (17\%) e 171 do sexo feminino $(83 \% ; \chi 2=89,79 ; p<0,001)$ e com uma idade média de idades de 18,83 anos $(D P=1,33)$. A maioria dos estudantes frequentava o curso de licenciatura $(97,1 \%)$, sendo que $74,8 \%$ destes cursava o $1 .^{\circ}$ ano, $12,1 \%$ o $2 .^{\circ}$ ano e $10,2 \%$ o $3 .^{\circ}$ ano do ensino superior. Relativamente às habilitações académicas dos pais dos participantes, somente $28,6 \%$ das mães possuíam habilitações académicas a nível superior, sendo esta percentagem inferior à dos pais dos participantes $(16,6 \%)$.

\section{Instrumentos}

Para a recolha de dados, foram aplicados três instrumentos. Para a caracterização da amostra procedeu-se à construção e aplicação de um questionário sociodemográfico no intuito de caracterizar os participantes sobre a idade, sexo, estado civil, habilitações e profissão dos pais, curso e ano frequentado e instituição de Ensino Superior frequentada.
Para avaliar a adaptação ao ensino superior recorreuse ao Questionário de Adaptação ao Ensino Superior (QAES), construído e validado por Araújo e colaboradores (2014). Este instrumento é constituído por 68 itens de autorresposta, tipo Likert com cinco opções de resposta (1 - Discordo Totalmente, 2 - Discordo, 3 Não discordo nem concordo; 4 - Concordo e 5 Concordo totalmente) e avalia seis dimensões independentes na adaptação ao ensino superior com bons índices de consistência interna: adaptação interpessoal ( $\alpha$ de Cronbach $=0,92$ ), adaptação à instituição $(\alpha$ de Cronbach $=0,72)$, adaptação académica ( $\alpha$ de Cronbach $=0,83$ ), compromisso com o curso ( $\alpha$ de Cronbach $=0,92$ ), adaptação pessoalemocional ( $\alpha$ de Cronbach $=0,91$ ) e desenvolvimento de carreira ( $\alpha$ de Cronbach $=0,92$ ).

Por último, para avaliar a autoconsciência da aparência aplicou-se a versão portuguesa reduzida da Escala de Avaliação de Derriford (DAS-14). Trata-se de uma versão reduzida do instrumento validado para a população portuguesa por Mendes e Pereira (2018), sendo constituído por um total de 14 itens, enquadrados numa escala tipo Likert, às quais se acrescentam quatro questões adicionais em torno da existência de algum aspeto/característica da aparência que gere preocupação: uma questão relativa ao aspeto sensível da aparência; uma questão que aborda o indivíduo não gosta na aparência e duas questões que focam outras características que influenciam a preocupação na aparência. Os 14 itens têm o objetivo de avaliar a forma como o indivíduo se sente e se comporta relativamente ao aspeto que o incomoda, sendo cotados através de dois conjuntos de resposta com cinco opções $(1$ - Nada, 2 - Ligeiramente, 3 Moderadamente, e 4 - Extremamente, ou de 0 - N/A (não aplicável); 1 - Nunca/Quase nunca, 2 - Às vezes, 3 Frequentemente, e 4 - Quase Sempre), não apresentando itens invertidos. A DAS-14 apresenta um bom índice de consistência interna ( $\alpha$ de Cronbach $=0,91$ ).

\section{Análise Estatística}

Para o tratamento estatístico foi utilizado o software Statical Package for the Social Sciences (SPSS), versão 22 para macOS. A análise da consistência interna dos instrumentos foi efetuada através do Alfa de Cronbach. Procedeu-se à correlação de Pearson para analisar possíveis associações entre as diversas variáveis, tendose prosseguido à comparação dos valores médios das variáveis em estudo através do teste $t$-Student $\mathrm{e}$ respetivos tamanhos do efeito (TDE). Por último, realizou-se uma análise de regressão linear múltipla com a finalidade de analisar o conjunto de variáveis que melhor predizem a adaptação ao ensino superior e a autoconsciência da aparência. 


\section{RESULTADOS}

Determinou-se a fiabilidade da escala QAES, obtendo-se um alfa de Cronbach de 0,91 para o total dos itens e valores igualmente adequados para as dimensões (Adaptação Interpessoal: $\alpha$ de Cronbach = 0,87; Adaptação à Instituição: $\alpha$ de Cronbach $=0,77$; Adaptação Académica: $\alpha$ de Cronbach $=0,83$; dimensão Compromisso com o Curso: $\alpha$ de Cronbach $=0,94$; Adaptação Pessoal-Emocional: $\alpha$ de Cronbach $=0,91$; Desenvolvimento de Carreira: $\alpha$ de Cronbach $=0,85$ ). A DAS-14 apresentou uma fiabilidade elevada $(\alpha$ de Cronbach $=0,91$ ).
Quanto à homogeneidade dos instrumentos, o QAES apresenta um Kaiser-Meyer-Olkin (KMO) de 0,90 $\left(\chi^{2}(9373,45) ; p \leq 0,001\right)$ e a DAS-14 apresentou um $K M O$ de $0,93\left(\chi^{2}(1345,31) ; p \leq 0,001\right)$.

Verificaram-se diferenças estatisticamente significativas na variável autoconsciência da aparência em função do sexo (Tabela 1), sendo a média superior no sexo feminino, com um TDE médio ( $g$ de Hedges $=0,57$ ).

Quanto à QAES, não se verificaram diferenças significativas quer para o total quer para as dimensões $(p$ $>0,05$ ) apresentado um TDE considerado pequeno ( $g$ de Hedges $<0,20$ ).

Tabela 1

Diferenças da Autoconsciência da Aparência (Escala de Avaliação de Derriford) Entre os Dois Sexos ( $\mathrm{N}=206)$

\begin{tabular}{|c|c|c|c|c|c|c|c|c|}
\hline \multirow{2}{*}{ Variáveis } & \multirow{2}{*}{ Sexos } & \multirow{2}{*}{$N$} & \multirow{2}{*}{ M } & \multirow{2}{*}{$D P$} & \multirow{2}{*}{$t$} & \multirow{2}{*}{$p$} & \multicolumn{2}{|c|}{$95 \% / C$} \\
\hline & & & & & & & Inferior & Superior \\
\hline \multirow{2}{*}{ Autoconsciência da Aparência } & Masculino & 35 & 18,46 & 8,14 & \multirow{2}{*}{$-3,05$} & \multirow{2}{*}{$<0,001$} & \multirow{2}{*}{$-9,23$} & \multirow{2}{*}{$-1,98$} \\
\hline & Feminino & 171 & 24,06 & 10,23 & & & & \\
\hline
\end{tabular}

Nota. $N=$ número de sujeitos; $M=$ Médias; $D P=$ Desvios-padrão; $t=$ teste $t$ de Student; $p=$ valor de significância; $I C=$ Intervalo de confiança.

A Tabela 2 apresenta resultados que indicam uma relação fraca, mas significativa entre a variável sexo e a Autoconsciência da Aparência. Esta apresenta uma correlação moderada e significativa com a dimensão Adaptação Pessoal-Emocional e uma relação negativa fraca, mas significativa com as restantes dimensões (Adaptação Interpessoal, Adaptação à Instituição, Adaptação Académica, Compromisso com o Curso e
Desenvolvimento de Carreira). Isto é, quanto menor Autoconsciência da Aparência maior a Adaptação Interpessoal, Adaptação à Instituição, Adaptação Académica e Compromisso com o Curso e Desenvolvimento de Carreira. Verificou-se, igualmente, uma relação positiva entre a Adaptação Académica e o Compromisso com o Curso $(r=0,51)$ e a Adaptação à Instituição e o Compromisso com o Curso $(r=0,41)$.

Tabela 2

Correlações Bivariadas entre o Sexo, a Autoconsciência da Aparência (Escala de Avaliação de Derriford - DAS-14) e as Dimensões do Questionário de Adaptação ao Ensino Superior (QAES) $(\mathrm{N}=206)$

\begin{tabular}{|c|c|c|c|c|c|c|c|c|}
\hline & 1 & 2 & 3 & 4 & 5 & 6 & 7 & 8 \\
\hline 1. Sexo & - & $0,21 * *$ & 0,19 & $-0,05$ & 0,05 & 0,01 & 0,10 & 0,02 \\
\hline 2. Autoconsciência da Aparência (DAS-14) & & - & $-0,18^{* *}$ & $-0,17^{*}$ & $-0,19 * *$ & $-0,15^{*}$ & $0,53 * *$ & $-0,14^{*}$ \\
\hline 3. Adaptação Interpessoal (QAES) & & & - & $0,35^{* *}$ & $0,25^{* *}$ & $0,39 * *$ & $-0,28^{* *}$ & $0,35^{* *}$ \\
\hline 4. Adaptação à Instituição (QAES) & & & & - & $0,37^{* *}$ & $0,41^{* *}$ & $-0,35 * *$ & $0,50 * *$ \\
\hline 5. Adaptação Académica (QAES) & & & & & - & $0,51 * *$ & $-0,30 * *$ & $0,55^{* *}$ \\
\hline 6. Compromisso com o Curso (QAES) & & & & & & - & $-0,33^{* *}$ & $0,62^{* *}$ \\
\hline 7. Adaptação Pessoal e Emocional (QAES) & & & & & & & - & $-0,33$ \\
\hline 8. Desenvolvimento de Carreira (QAES) & & & & & & & & - \\
\hline M & 1,83 & 23,11 & 3,75 & 3,75 & 3,41 & 3,83 & 3,16 & 3,16 \\
\hline$D P$ & 0,38 & 10,11 & 0,66 & 0,64 & 0,69 & 0,78 & 0,91 & 0,76 \\
\hline
\end{tabular}

$* p<0,05 ; * p<0,01$. 
O modelo de regressão linear múltipla da Autoconsciência da Aparência em função das dimensões da adaptação ao ensino superior, revelou-se estatisticamente significativo $\left(F(6,199)=13,50 ; R^{2}=0,30\right.$; $p \leq 0,001)$, tendo-se revelado a dimensão Adaptação Pessoal-Emocional como preditora da Autoconsciência da Aparência $(\beta=0,53 ; t(199)=8,01 ; p \leq 0,001)$.
Noutro sentido, analisarem-se as dimensões da adaptação ao ensino superior, em função da variável Autoconsciência da Aparência, o modelo revelou-se estatisticamente significativo em todas as dimensões do QAES (Tabela 3), observando-se maior variabilidade entre a variável Autoconsciência da Aparência e Adaptação pessoal-emocional $(27,8 \%)$.

Tabela 3

Regressão Linear Múltipla Utilizando a Variável Autoconsciência da Aparência Para Predizer As Dimensões do Questionário de Adaptação ao Ensino Superior (QAES) (N = 206)

\begin{tabular}{lcccccc}
\hline \multicolumn{7}{c}{ Autoconsciência da Aparência } \\
\hline Dimensões do QAES & $B$ & $\beta$ & $t$ & $p$ & $F(g l)$ & $R^{2}$ \\
\hline Adaptação interpessoal & $-0,012$ & $-0,184$ & $-2,67$ & 0,008 & $7,12(204)$ & 0,029 \\
Adaptação à instituição & $-0,011$ & $-0,172$ & $-2,49$ & 0,014 & $6,18(204)$ & 0,025 \\
Adaptação académica & $-0,013$ & $-0,185$ & $-2,69$ & 0,008 & $7,22(204)$ & 0,029 \\
Compromisso com o curso & $-0,011$ & $-0,146$ & $-2,103$ & 0,037 & $4,43(204)$ & 0,016 \\
Adaptação pessoal-emocional & 0,048 & 0,53 & 8,93 & $<0,001$ & $79,87(204)$ & 0,278 \\
Desenvolvimento de carreira & $-0,010$ & $-0,139$ & $-2,01$ & 0,046 & $4,02(204)$ & 0,015 \\
\hline
\end{tabular}

Nota. $g l=$ graus de liberdade.

\section{DISCUSSÃO}

Pretendeu-se com o presente estudo analisar possíveis relações entre as preocupações com a aparência e a adaptação ao Ensino Superior. Os resultados obtidos apontam para uma boa fiabilidade e homogeneidade das variáveis, tendo-se obtido valores de alfa de Cronbach e Kaiser-Meyer-Olkin considerados bons (Marôco, 2010). Apesar dos resultados apontarem para diferenças estatisticamente significativas na Autoconsciência da Aparência segundo o sexo dos estudantes, temos de ter em conta que a amostra é significativamente maior no sexo feminino, apresentando diferenças significativas na variável autoconsciência da aparência em relação ao sexo masculino. Contudo, os resultados não verificaram diferenças significativas nas dimensões do QAES, apresentando tamanhos do efeito considerados pequenos (Espírito-Santo \& Daniel, 2015). Um estudo de Medeiros et al. (2018) com estudantes universitários verificou que o sexo feminino apresenta maiores níveis de preocupação com a forma corporal. Estes resultados podem estar relacionados com o facto de as mulheres compararem a aparência entre si, influenciando a sua imagem corporal (Fardouly et al., 2017), ou estarem mais preocupadas com a sua adaptação interpessoal (Almeida, Guisande, Soares, \& Saavedra, 2006).
Verificam-se fracas relações negativas, mas significativas entre a Autoconsciência da Aparência e as dimensões de Adaptação à Ensino Superior, à exceção da dimensão Adaptação Pessoal-Emocional que apresenta uma correlação positiva moderada e também significativa. A dimensão Adaptação Pessoal-Emocional revelou-se ainda como variável preditora da Autoconsciência da Aparência. $\mathrm{Na}$ verdade, os resultados parecem evidenciar uma tendência para que uma menor autoconsciência da aparência (menos sentimentos negativos em torno do aspeto do corpo) se relacione com uma maior centração nas questões de adaptação académica (curso, instituição e carreira) e nas questões interpessoais, num desenvolvimento mais positivo e construtivo. Estes resultados podem dever-se ao facto de os adultos emergentes que ingressam no ensino superior estarem numa fase de transição, numa nova etapa na construção de identidade e do desenvolvimento das relações interpessoais, onde influenciam e são influenciados pelos grupos em que se inserem (Almeida et al., 2006; Araújo, Costa, Casanova, \& Almeida, 2014; Araújo et al., 2016; Costa, Araújo, Diniz, \& Almeida, 2014; Nunes \& Garcia, 2010), estando confortáveis com a sua aparência (Tomás, Ferreira, Araújo, \& Almeida, 2014).

Salienta-se que a construção da identidade social está fortemente associada à perceção e funcionamento do corpo (Santos \& Silva, 2017), existindo uma associação 
entre a imagem e as representações relacionadas com o corpo (Moliner, 2016), influenciando as dificuldades de relacionamento interpessoal quando existe uma insatisfação com a imagem corporal (Garcia, Macedo, \& Nunes, 2013). Estudos indicam que a construção da identidade pode estar associada aos primeiros dois anos de frequência do ensino superior (Araújo et al., 2016; Costa et al., 2014), no entanto, não se verificaram diferenças estatisticamente ou correlação entre o ano de frequência do ensino superior e a autoconsciência da aparência, uma vez que a maioria dos estudantes se encontrava a frequentar o primeiro ano do Ensino Superior.

\section{Conclusão}

O nosso estudo apresenta limitações que devem ser realçadas, incluindo o facto de a amostra apresentar uma desproporção quanto aos sexos e não se ter explorado a altura e o peso dos participantes de forma a explorar possíveis relações entre o índice de massa corporal e autoconsciência da aparência.

No entanto, os instrumentos apresentam bons índices de fiabilidade e homogeneidade e a amostra apresentou uma dimensão adequada para as análises estatísticas efetuadas. Em síntese, o sexo feminino apresenta maior preocupação com a aparência em relação ao sexo masculino. Verificou-se uma fraca relação entre a autoconsciência da aparência (sentimentos negativos em torno do aspeto do corpo) e as dimensões da adaptação ao ensino superior, podendo inferir-se que os estudantes que não tem tendência à desvalorização da sua aparência concentram-se mais nas dimensões sociais, cognitivas e contextuais da adaptação ao ensino superior. A autoconsciência da aparência apresenta-se neste estudo como possível variável preditora das dimensões da adaptação ao ensino superior.

A presente investigação permite concluir que as preocupações com a aparência e a adaptação pessoalemocional se influenciam mutuamente, isto é, a aceitação da aparência parece ser relevante para a adaptação e para o bom desenvolvimento da identidade dos estudantes do ensino superior.

Sendo desconhecidos estudos que analisem as preocupações com a aparência como variável preditora, consideramos a contribuição desta investigação como pioneira no estudo sobre a autoconsciência da aparência, recomendando-se mais investigações nesta área da Psicologia da Aparência e nas suas relações com o desenvolvimento positivo e a saúde.
Conflito de interesses | Conflict of interest: Nenhum | None.

Fontes de financiamento | Funding sources: Nenhuma | None.

\section{REFERÊNCIAS}

Almeida, L. S., Guisande, M. A., Soares, A. P., \& Saavedra, L. (2006). Acesso e sucesso no ensino superior em Portugal: Questões de género, origem sócio-cultural e percurso académico dos alunos [Access and sucess in higher education in Portugal: Issues of gender, sociocultural origin and students' academic path]. Psicologia: Reflexão e Crítica, 19(3), 507-514. https://doi.org/10.1590/S0102-79722006000300020

Araújo, A. M., Almeida, L. S., Ferreira, J. A. G., Santos, A. A., Noronha, A. P., \& Zanon, C. (2014). Questionário de Adaptação ao Ensino Superior (QAES): Construção e validação de um novo questionário [Adaptation to Higher Education Questionnaire (QAES): Construction and validation of a new questionnaire]. Psicologia, Educação e Cultura, 18(1), 131-145. Retrieved from http://pec.ispgaya.pt/edicoes/2014/PEC2014N1/index.html

Araújo, A. M., Costa, A. R., Casanova, J. R., \& Almeida, L. S. (2014). Questionário de Perceções Académicas - Expetativas: Contributos para a sua validação interna e externa [Academic Perceptions - Expectations Questionnaire: Contributions for internal and external validation]. Revista E-Psi, 1(4), 156-178. Retrieved from https://revistaepsi.com/wpcontent/uploads/artigos/2014/Ano4-Volume1-Artigo8.pdf

Araújo, A. M., Santos, A. A., Noronha, A. P., Zanon, C., Ferreira, J. A., Casanova, J. R., \& Almeida, L. S. (2016). Dificuldades antecipadas de adaptação ao ensino superior: Um estudo com alunos do primeiro ano [Anticipated adaptation difficulties to higer education: A study with first-year students]. Revista de Estudios e Investigación en Psicología y Educación, 3(2), 102-111. https://doi.org/10.17979/reipe.2016.3.2.1846

Arnett, J. J. (2004). Emerging adulthood: The winding road from the late teens through the twenties (1st ed.). Oxford: University Press.

Behmani, R. K., \& Kumar, S. (2016). Gender and locality differences in body image among college students. Indian Journal of Community Psychology, 12(1), 130-138. Retrieved from http://www.ijcpind.com/wpcontent/uploads/Website\%20versonlJCP,2016(1).pdf

Carr, T., Harris, D., \& James, C. (2000). The Derriford Appearance Scale (DAS-59): A new scale to measure individual responses to living with problems of appearance. British Journal of Health Psychology, 5(2), 201-215. https://doi.org/10.1348/135910700168865

Carr, T., Moss, T., \& Harris, D. (2005). The DAS24: A short form of the Derriford Appearance Scale DAS59 to measure individual responses to living with problems of appearance. British Journal of Health Psychology, 10(2), 285-298. https://doi.org/10.1348/135910705X27613

Cash, T. F., \& Pruzinsky, T. (2002). Body image: A handbook of theory, research, and clinical practice (1st ed.). New York, NY: Guilford Press.

Cash, T. F. (2002). A «negative body image»: Evaluating epidemiological evidence. In T. F. Cash \& T. Pruzinsky (Eds.), Body Image: A handbook of theory, research, and clinical practice (1st ed., pp. 269-276). New York, NY: Guilford Press.

Cash, T. F., \& Smolak, L. (2011). Body Image: A handbook of science, practice, and prevention (2nd ed.). New York, NY: Guilford Press.

Costa, A. R., Araújo, A. M., Diniz, A. M., \& Almeida, L. S. (2014). Expetativas académicas segundo o género dos estudantes portugueses do primeiro ano do ensino superior. International Journal of Developmental and Educational Psychology, 2(1), 325334. https://doi.org/10.17060/ijodaep.2014.n1.v2.447

Deaño, M. D., Gil, S. A., Rodríguez, Á. C., García-Señorán, M., \& González, F. T. (2014). Expectativas universitarias y predicción del rendimiento académico [University expectations and academic performance prediction]. Revista E-Psi, 1(4), 127-141. Retrieved from https://revistaepsi.com/wpcontent/uploads/artigos/2014/Ano4-Volume1-Artigo6.pdf 
Espírito-Santo, H., \& Daniel, F. (2015). Calcular e apresentar tamanhos do efeito em trabalhos científicos (1): As limitações do $p<0,05$ na análise de diferenças de médias de dois grupos [Calculating and reporting effect sizes on scientific papers (1): $p<0.05$ limitations in the analysis of mean differences of two groups]. Revista Portuguesa de Investigação Comportamental e Social, 1(1), 3-16. https://doi.org/10.7342/ismt.rpics.2015.1.1.14

Fardouly, J., Pinkus, R. T., \& Vartanian, L. R. (2017). The impact of appearance comparisons made through social media, traditional media, and in person in women's everyday lives. Body Image, 20, 31-39. https://doi.org/10.1016/j.bodyim.2016.11.002

Freitas, H. C. N. M., Raposo, N. A. V., \& Almeida, L. S. (2007). Adaptação do estudante ao ensino superior e rendimento académico: Um estudo com estudantes do primeiro ano de enfermagem. Revista Portuguesa de Pedagogia, 41(1), 179-188. Retrieved from http://impactumjournals.uc.pt/rppedagogia/article/view/1191

Garcia, A., Macedo, M. D. C., \& Nunes, T. A. (2013). Relações interpessoais e saúde [Interpersonal relations and health] (1st ed.). Vitória: UFES

Latiff, A. A., Muhamad, J., \& Rahman, R. A. (2018). Body image dissatisfaction and its determinants among young primary-school adolescents. Journal of Taibah University Medical Sciences, 13(1), 34-41. https://doi.org/10.1016/j.jtumed.2017.07.003

Lopes, M. S., Mendes, R. C. P. R., \& Sousa, S. M. N. (2017). Ser mulher: Uma análise da imagem corporal entre adolescentes. Revista Espacios, 38(29). Retrieved from http://www.revistaespacios.com/a17v38n29/a17v38n29p03.pdf

MacKenzie, K. (2012). An examination of coping strategies and mindfulness as predictors of appearance satisfaction and body image. (Master's thesis). Retrieved from https://dra.american.edu/islandora/object/thesesdissertations $\% 3 \mathrm{~A}$ 91/datastream/PDF/view

Marôco, J. (2010). Análise estatística com o PASW Statistics (ex-SPSS). Lisboa: ReportNumber.

Martins, D. F., Nunes, M. F. O., \& Noronha, A. P. P. (2008). Satisfação com a imagem corporal e autoconceito em adolescentes. Psicologia: Teoria e Prática, 10(2), 94-105.

Medeiros, T., Cunha, A., Lima, C., Ponte, V., Furtado, S., \& Mendes, J. C. S. (2018). Imagem corporal positiva em adultos emergentes: Um estudo em contexto universitário [Positive body image in emergente adults: A study in university context] (pp. 813-821). In Actas do $12^{\circ}$ Congresso Nacional de Psicologia da Saúde. Lisboa: ISPA - Instituto Universitário.
Mendes, J., \& Pereira, V. (2018). Versão portuguesa reduzida da Escala de Avaliação da Aparência de Derriford (DAS-14): Análise fatorial exploratória e confirmatória. Revista Portuguesa de Investigação Comportamental e Social, 4(2), 23-30. https://doi.org/10.31211/ismt.rpics.2018.4.2.79

Moliner, P. (2016). Psychologie sociale de l'image [Social psychology of the image]. Fontaine: PUG.

Moss, T. P., \& Rosser, B. A. (2012). The moderated relationship of appearance valence on appearance self-consciousness: Development and testing of new measures of appearance schema components. PLOS ONE, 7(11), e50605. https://doi.org/10.1371/journal.pone.0050605

Nunes, S. M., \& Garcia, A. R. (2010). Estudantes do ensino superior: As relações pessoais e interpessoais nas vivências académicas. Gestin: Revista Científica da Escola Superior de Gestão, 8(8), 195-203. Retrieved from http://gestin.ipcb.pt/index.php/gestinviii

Rodrigues, D., Teves, C., \& Medeiros, T. (2013). Autoimagem e satisfação corporal na adolescência [Self-image and body satisfaction in adolescence]. In T. Medeiros (Coord.), Adolescência: Desafios e riscos (Chapter 8; pp. 183208). Ponta Delgada: Letras Lavadas.

Rumsey, N., \& Harcourt, D. (2012). The Oxford handbook of the psychology of appearance. Oxford: Oxford University Press.

Santos, O. S., \& Silva, A. (2017). Corpo, deficiência e representações. Revista Educação e Cultura Contemporânea, 14(37), 446-464. https://doi.org/10.5935/2238-1279.20170056

Smolak, L. (2012). Appearance in chilhood and adolescence. In N. Rumsey \& D. Harcourt (Eds.), The Oxford handbook of the psychology of appearance (pp. 123-141). Oxford: Oxford University Press.

Souza, A. C., \& Alvarenga, M. S. (2016). Insatisfação com a imagem corporal em estudantes universitários - Uma revisão integrativa. Jornal Brasileiro de Psiquiatria, 65(3), 286-299. https://doi.org/10.1590/0047-2085000000134

Tiggemann, M. (2015). Considerations of positive body image across various social identities and special populations. Body Image, 14, 168-176. https://doi.org/10.1016/j.bodyim.2015.03.002

Tomás, R. A., Ferreira, J. A., Araújo, A. M., \& Almeida, L. S. (2014). Adaptação pessoal e emocional em contexto universitário: $\mathrm{O}$ Contributo da personalidade, suporte social e inteligência emocional [Personal and emotional adaptation in University's environment: Contribution of personality, social support and emotional intelligence]. Revista Portuguesa de Pedagogia, 48(2), 87-107. https://doi.org/10.14195/1647-8614_48-2_5

Vieira, E. T., \& Grantham, S. (2011). University students setting goals in the context of autonomy, self-efficacy and important goal-related task engagement. Educational Psychology, 31(2), 141-156. https://doi.org/10.1080/01443410.2010.536508 